\title{
Energy Harvesting Embedded Wireless Sensor System for Building Environment Applications
}

\author{
W. S. Wang ${ }^{1}$, T. O’Donnell ${ }^{1}$, L. Ribetto ${ }^{2}$, B. O’Flynn ${ }^{1}$, M. Hayes ${ }^{1}$, C. O’Mathuna ${ }^{1}$ \\ ${ }^{1}$ Tyndall National Institute, Microelectronics Applications Integration Group, Cork, Ireland \\ ${ }^{2}$ Ecole Polytechnique Fédérale de Lausanne (EPFL), Sensors Actuators and Microsystems Laboratory, Switzerland
}

wensi.wang@tyndal1.ie

\begin{abstract}
For many wireless sensor networks applications, indoor light energy is the only ambient energy source commonly available. Many advantages and constraints co-exist in this technology. However, relatively few indoor light powered harvesters have been presented and much research remains to be carried out on a variety of related design considerations and trade-offs. This work presents a solution using the Tyndall mote and an indoor light powered wireless sensor node. It analyses design considerations on several issues such as indoor light characteristics, solar panel component choice, maximum power point tracking, energy storage elements and the trade-offs and choices between them.
\end{abstract}

Keywords- Light energy harvesting; Wireless sensor networks; MPPT; Energy storage

\section{INTRODUCTION}

In the last decade, the miniaturization and functional improvement of ICs has led to a prolifiteration of wireless sensor network (WSN) applications. As a result, several WSN products have already been deployed in volume in commercial applications [1,2].

The optimized network algorithm and higher functional complexity of the wireless sensor nodes may enable WSN systems replace most of the current 'wired' (cable-connected) sensor systems in the foreseeable future.

In building environmental management (BEM) applications area, the demand for WSN technology has increased in recent years. For example, about $40 \%$ of Ireland's energy usage and over $30 \%$ of $\mathrm{CO} 2$ emissions can be attributed to the running of residential and commercial buildings. The usage of intelligent WSNs can result in $15-20 \%$ savings in energy usage [3]. Due to such potential financial benefits and the political 'green agenda', intensive research interests have been attracted to this area. For such applications the core considerations are monitoring of artificial lighting, temperature, $\mathrm{CO} 2$ level, relative humidity, positioning of external shading devices and resultant actuation required.

However, one major bottleneck for all WSN deployments has been the limited lifetime due to the insufficient energy capacity of the battery power supply. For example, a Tyndall Institute designed [8] wireless sensor node with temperature and relative humidity sensor consume an average $350 \mathrm{uW}$ power when it operates on a $0.1 \%$ duty cycle. Operating with two standard $3000 \mathrm{mAh}$ AA battery cells at first glance indicates the requirement for battery replacement every 200 days. In practicality, however, the battery life time is even shorter due to leakage current, temperature factors, environmental humidity and other variable factors [4]. With the increasing deployment scale of wireless sensor nodes in WSN systems, the market demands a 'deploy and forget' solution requiring the elimination of a battery replacement maintenance cycle. Energy harvesting technology could lead to this possibility of self-sustaining infinite lifetime motes, or at least the prolongation on the life span of the system between battery replacements. This is a significant focus area in WSN research in recent years because of the necessity to bridging the gap between the continuous power consumption of the mote and the limited available energy from the current battery technology.

Several types of energy sources are available to be harvested in the ambient environment. Light irradiance, temperature gradient and vibration energy have drawn the most attention within the research community. Some research shows a detailed comparison of energy density between different energy sources [5, 6]. Among these energy scavenging techniques, light energy harvesting is a relatively mature technology and light energy is one of the most ubiquitous ambient energy types. Typical applications using solar energy as a power source are focussed in the outdoor environment, where the variation of the ambient light energy source is regular and relatively predictable. The commercial availability of photovoltaic panels is also better than piezoelectric generators and thermoelectric generators. Therefore, this paper focuses on energy harvesting from indoor light energy designated for BEM. For indoor application, the overall light energy density is inherently limited. A typical light energy density in a fluorescent lighting condition (500lux) is approximately $185 \mathrm{uW} / \mathrm{cm}^{2}$ or one to two orders lower than the outdoor light energy density [7]. This together with the limited light energy conversion efficiency in indoor environment and the natural variability of the light energy make indoor light energy harvesting very challenging. It is important to design the energy harvesting system so as to guarantee it can handle a wide range of input light energy densities and have high energy conversion efficiency from the available energy sources. In addition, many other system design issues require consideration such as small form factor, output voltage level, capacity of the energy storage, cost efficiency and 'plug and play' ability. Furthermore, these factors are not isolated, but are dynamically inter-related. However, compared to well addressed outdoor macro-power systems, there is no significant evidence of thorough studies on these design issues and the trade-offs between them. 
This paper addresses the design considerations of an indoor light energy harvesting devices for BEM applications. Firstly, the Tyndall $25 \mathrm{~mm}$ wireless sensor system is introduced and the design requirements and system architecture of the proposed indoor light energy harvester discussed. Then the characteristics of the indoor light energy sources are analysed and the choice of the solar panel based on these characteristics reviewed. Optimization of the power management is considered based on various maximum power point tracking approaches. Storage elements and several trade-offs on this issue are outlined. Based on these considerations, a design solution of the indoor solar panel embedded wireless sensor system is presented. Finally, operation results of the energy harvesting devices are shown and performance evaluated.

\section{TYNDALL WIRELESS SENSOR NODE}

To design an efficient energy harvester, an analysis on its load, the wireless sensor node in operation, is essential. In this section, the amount of energy consumption of the wireless sensor node and its energy consumption pattern is presented. The analysis is based on the experimental results of the Tyndall mote, as illustrated in Figure 1. The Tyndall mote has a multiple layer design with extension/replacement ability and can be configured to take different power layers and sensor layers with only minor changes on hardware and software [8].

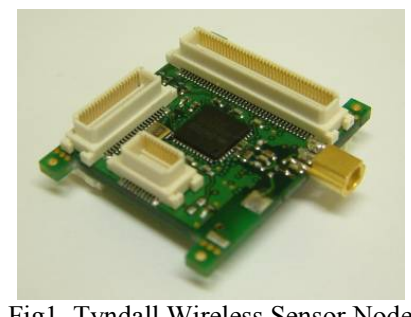

Fig1. Tyndall Wireless Sensor Node

In general, the wireless sensor node is a microcontroller controlled sensing and communication module. During its sensing and transmitting/receiving mode, much higher power is consumed than in sleep mode, the active power consumption being 1863 times higher than its average sleep mode power consumption as shown in Table I. As a result of this, most wireless sensor nodes adopt an asymmetric ratio duty cycling operation.

Table I. Tyndall Mote Power Consumption

\begin{tabular}{|l|l|l|l|}
\hline & $\begin{array}{l}\text { Power } \\
\text { Consumption }(\mathrm{mW})\end{array}$ & $\begin{array}{l}\text { Time } \\
(\mathrm{sec})\end{array}$ & $\begin{array}{l}\text { Ratio Duty } \\
\text { Cycle (\%) }\end{array}$ \\
\hline Active & 111.90 & 0.039 & 0.065 \\
\hline Sleep & 0.0601 & 59.961 & 99.935 \\
\hline Average & 0.1326 & 60 & 100 \\
\hline
\end{tabular}

The overall power consumption of the wireless sensor node, therefore, highly depends on the duty cycle. A simple equation can be used to estimate the overall power consumption, where $\rho$ is the active mode duty cycle ratio.

$$
P_{\text {average }}=P_{\text {active }} \rho+P_{\text {sleep }} \cdot(1-\rho)
$$

Evidently there is a trade-off between the power consumption, system data process capability and programmability. The Tyndall mote's design strategy is geared towards energy efficiency, giving relatively smaller power consumption when compared to other generic function motes, but has higher consumption than application-specified motes $[9,10]$.In the proposed BEM system, two types of measurement interval are required; namely one minute per measurement and 15 minutes per measurement intervals. The requirements result in different power consumption. For 1 minute intervals, the energy consumption in one hour operation is $339 \mathrm{~mJ}$, but for 15 minutes per measurement, the energy consumption in one hour operation is only $169 \mathrm{~mJ}$. It is worth to note that the sleep mode consumes a large portion of the total energy as shown in Figure 2, measuring 46\% (1 minute intervals) and $92 \%$ (15 minutes intervals) of the total energy respectively.
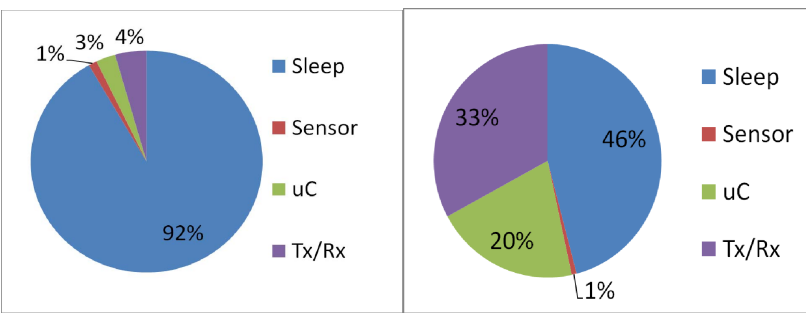

Fig2. Tyndall Wireless Sensor Node Power Consumption Pie Chart

The WSN power consumption pattern shows two main features: (i) The power consumption is highly sensitive to the operation duty cycle. (ii) Sleep mode power consumption is critical for low duty cycle applications. These two features markedly affect the design considerations and system architecture of the energy harvester system. The effects are introduced in later sections.

\section{SYSTEM ARCHITECTURE}

The operational scenario of this energy harvester embedded WSN prototype is in a typical office environment. The application is to quantify the environment parameters and reduce the daily energy consumption of the building through a WSN based building management system. The proposed WSN mote consists of five main components: the light available in the environment, the solar panel, energy management circuits, energy storage and wireless sensor node as shown in Figure 3.

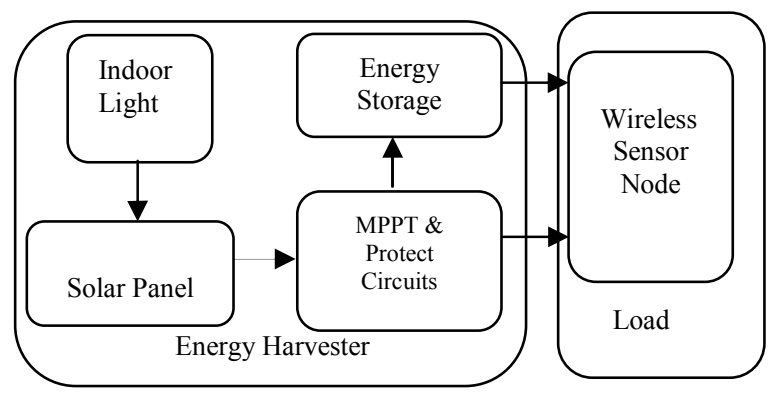

Fig3. System Architechture

The design goals of these components are: (i) to correctly analyse the indoor light energy characteristics (ii) to choose a high efficiency solar panel for indoor light (iii) to obtain an operation voltage close to the maximum power point (MPP) (iv) to choose an energy storage that can efficiently store the energy and deliver it to the load. These goals are considered as 
the design priorities on each issue, and will affect the overall energy conversion efficiency and the system performance.

\section{HARVESTER DESIGN CONSIDERATIONS}

This section discusses the characterizations, design considerations, limits and adaptability of available energy harvesting sources.

\section{A.. Indoor Light Energy}

Several special features should be considered when harvesting the indoor light energy. Firstly, indoor light energy has limited overall energy irradiance compared to outdoor light energy. The indoor illuminance level generally ranges from 100lux to 1000lux, or one order lower than the outdoor illuminance. Since the conversion efficiency of the solar panel also decreases with decreasing irradiance in low illuminance level, this results in an even lower converted energy [11].

Previous-work-on-indoor-light-energy-[16]-shows that for values of the incoming power density in the indoor environment, the efficiency achieved by the cells was typically between $3-10 \%$. This efficiency is typically $60 \%$ lower than the outdoor solar panel's conversion efficiency. Several typical irradiance levels and conversion efficiencies are shown in Table II for several common solar cell types, crystalline Silicon (c-Si), amorphous Silicon (a-Si) and GaAs $[12,13]$.

Table II. Typical environment irradiance level and solar panel energy conversion efficiency

\begin{tabular}{|l|l|l|l|}
\hline \multicolumn{2}{|l|}{} & $\begin{array}{l}\text { Indoor } \\
\text { Light }\end{array}$ & $\begin{array}{l}\text { Outdoor } \\
\text { Light }\end{array}$ \\
\hline Illuminance level (lux) & $\begin{array}{l}100- \\
10001 u x\end{array}$ & $\begin{array}{l}1000- \\
65,0001 u x\end{array}$ \\
\hline \multirow{2}{|l|}{$\begin{array}{l}\text { Solar Panel Energy } \\
\text { Conversion } \\
\text { Efficiency }\end{array}$} & $\mathrm{c}-\mathrm{Si}$ & $3-10 \%$ & $\sim 18 \%$ \\
\cline { 2 - 4 } & $\mathrm{a}-\mathrm{Si}$ & $2-5 \%$ & $8-13 \%$ \\
\cline { 2 - 4 } & $\mathrm{GaAs}$ & $2 \sim 8 \%$ & $7-15 \%$ \\
\hline
\end{tabular}

Secondly,- the- various- indoor- light- sources - irradiancegenerates- different- responses- on- the- solar- panels.- Thesame- illuminance- level,- when- generated- from- differentlight- sources,- results - in- different- levels- of- powergeneration,-since-the-spectra-of-light-sources-are-different,the- actual- irradiances- from- various- light- sources- ondifferent-wavelengths-of-light-are-different.--

Among- the- most-common- light- sources,- sunlight- andhalogen-lights-have-a-higher-power-spectral-density-(PSD) on-red-light-(near-656nm)-and-fluorescent-light-and-LEDlight- have- relatively- high- power- density- on- blue- lightwavelength- (near-550nm).- The- different-materials- andtechnologies- used- in- solar- cells- will- lead- to- differentspectral- responses- and- power- densities.- Figure- 4illustrates- the- differences of the power density measured on a GaAs and an amorphous Silicon solar panel when different light sources are used to generate the same illuminance level.

Several other issues will also largely affect the results of the light energy harvesting. These factors include: (i) the distance and the angle between the solar panel and the indoor light sources. (ii) Surface temperature of the solar panel.(iii) Limitations on the devices form factors.

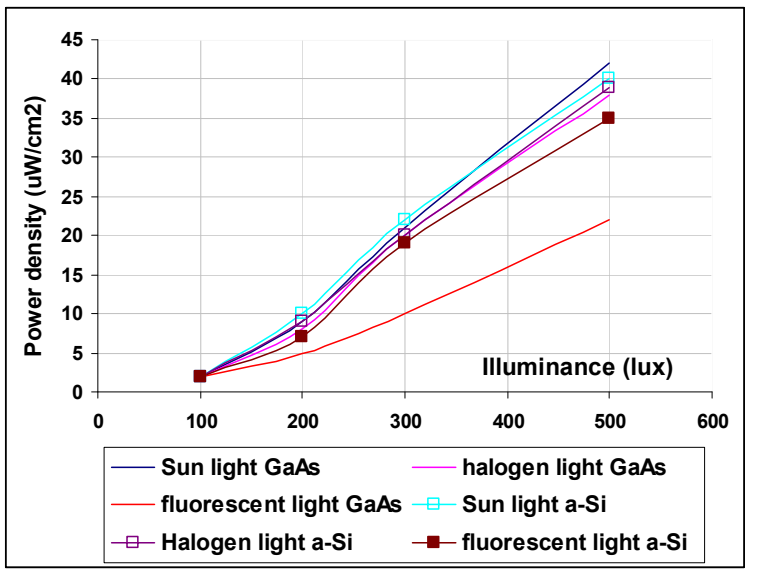

Fig.4 Power spectral densities of light sources at a constant illuminance

\section{B. Photovoltaic Characteristics}

Based on previous considerations on indoor light energy in the proceeding section, a solar panel with higher conversion efficiency in indoor environment and high response sensitivity on typical office indoor fluorescent light source's spectrum is selected.

The Schott Solar's ASI2Oi05/055/014JF's base material is amorphous Silicon deposited on a glass substrate; each cell is based on a-Si PIN technology. The active area of the module is $50 \mathrm{~mm} \times 13.5 \mathrm{~mm}$. The Solar panel's I-V characteristics and $\mathrm{P}-\mathrm{V}$ characteristics are shown in Figure 5. The maximum power under 100lux, 200lux and 500lux are $12.73 \mathrm{uW}$, $39.05 \mathrm{uW}$ and $151.59 \mathrm{uW}$, respectively. To supply sufficient power to the mote at 200lux illuminance, at least 4 cells are required.

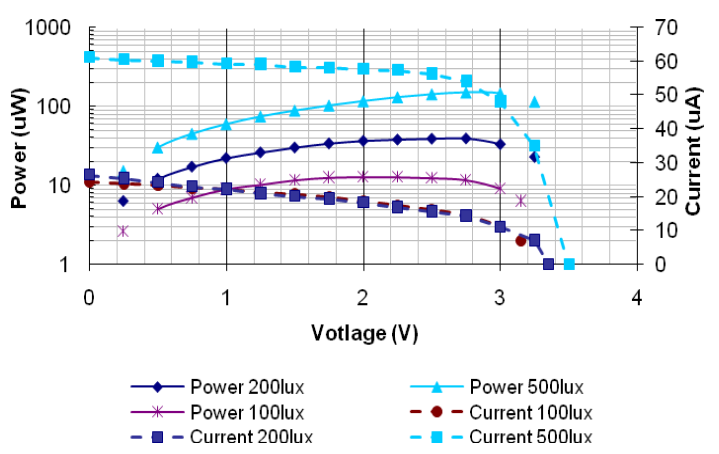

Fig.5 The I-V and P-V characteristics of the Schott Solar's ASI2Oi05/055/014JF

These IV characteristics show the solar panel has a similar behaviour to a voltage limited current source. The open circuit voltage is significantly less affected over different irradiance level than the short circuit current. The voltage limited current source-like IV characteristics show three features of the solar panel. (i) The maximum power point (MPP) is most likely be generated from high voltage level operation, due to the close to constant current output on lower voltage level. (ii) In lower illuminance level (200lux), a relatively wide voltage range operates close to maximum power point (MPP). (iii) Also, due to the current source behaviour, directly connecting and 
powering the wireless sensor system from the solar panel is infeasible and would greatly reduce the reliability of the system operation. An energy storage element is required to work as a buffer and temporary energy reservoir between the current source-like photovoltaic cell and the load.

\section{Maximum Power Point Tracking (MPPT) Issues}

To ensure the solar panel operates at close to maximum power point, a maximum power point tracking (MPPT) scheme is considered. The most commonly used MPPT approach is Perturb-and-Observe. This method uses a microcontroller or DSP to control a switching regulator. The switching regulator then controls the output voltage of the solar panel, and delivers the energy to the load. The Perturband-Observe method can accurately track the MPP, and the response time over abrupt changes on input is also short. To implement the Perturb-and-Observe approach, two core components, a control-unit (microcontroller or DSP) and a switching regulator, are essential. However, the major drawback of this MPPT system is the relatively high power consumption of these components. The power consumption of the MPPT circuit can be approximately calculated by using equation (2),

$$
P_{m p p t} \approx\left(I_{u c}+I_{s r}\right) \cdot V_{m p p}+I_{o s c} \cdot V_{o s c}
$$

where $\mathrm{I}_{\mathrm{uc}}, \mathrm{I}_{\mathrm{sr}}, \mathrm{I}_{\mathrm{osc}}$ are the current consumptions of microcontroller, switching regulator and crystal oscillator, respectively.

The MPPT scheme could be implemented by using a dc-dc converter such as the TI buck/boost converter [14], controlled by the Tyndall mote's ATmega128 microcontroller, and a 32 $\mathrm{KHz}$ low power oscillator. Using this scheme the MPPT tracker's power consumption is calculated at the level of $300 \mathrm{uW}$ [15]. Under 500 lux illuminance condition, the simulated operation voltage without MPPT is $3.14 \mathrm{~V}$; the output power is $129 \mathrm{uW}$. The maximum power point in this condition is $151.59 \mathrm{uW}$, as shown in Figure 6. Hence, for each Schott Solar cell, the operation power gains $22.6 \mathrm{uW}$ from the MPPT scheme. For 4 solar cells, the power gain from the MPPT is $90.4 \mathrm{uW}$. The power gain from the MPPT is therefore significantly less than the MPPT scheme power consumption $(300 \mathrm{uW})$.

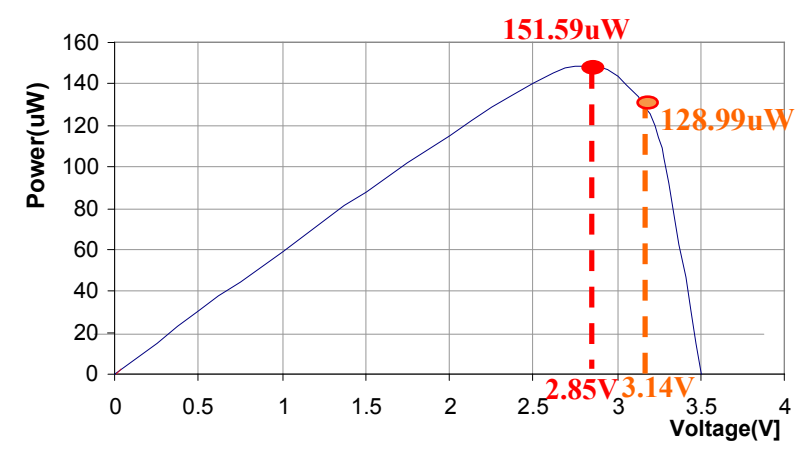

Fig.6 P-V characteristics of the Schott Solar panel and the MPP

Another simpler and less energy costly MPPT method is the fractional open circuit voltage approach. This method is based on the near-linear relation between the maximum power point operation voltage $\mathrm{V}_{\mathrm{mpp}}$ and the open circuit voltage $\mathrm{V}_{\mathrm{oc}}$. The relationship between these two can be described in Equation (3).

$$
V_{m p p}=V_{o c} \cdot K_{f v o c}
$$

Some research considers the $\mathrm{k}_{\mathrm{fvoc}}$ as a constant ranging from 0.71 to 0.78 , depending on the photovoltaic panel's material characteristics and environment irradiance conditions. Other research asserts that the $\mathrm{k}_{\mathrm{fvoc}}$ could simply considered as a constant 0.74 , with a $+/-5 \%$ error in maximum power point voltage evaluation [16]. Based on this, a reference voltage from a secondary battery and a low power LDO can preset the voltage to $\mathrm{k}_{\mathrm{fvoc}} * \mathrm{~V}_{\mathrm{oc}}$. A hysteresis voltage comparator can be used as a voltage follower to allow the operation voltage approximately equal to theoretical maximum power point voltage $\mathrm{k}_{\mathrm{fvoc}}{ }^{*} \mathrm{~V}_{\mathrm{oc}}$. This MPPT approach significantly reduces the power consumption; A MPPT scheme implemented using this method, with an ultra low power comparator, a MOSFET switch and an LDO is estimated to consume only around $30 \mathrm{uW}$.

However, the trade-off on the accuracy of the maximum power point voltage is also considerable. And furthermore, in lower irradiance level, the error of the constant $\mathrm{k}_{\mathrm{fvoc}}$ differs from the previous value. The ratio between maximum power point voltage and the open circuit voltage of the amorphous silicon based Schott solar panel under 500lux, 200lux and 100lux fluorescent light it measured at $0.81,0.82$ and 0.79 respectively; it differs from the claimed $0.74+/-5 \%$ range. Therefore, when implementing this MPPT method, a significant drift from the actual MPP is expected. The $V_{\text {mpp }}$ calculated by using equation (3) is $2.59 \mathrm{~V}$; and the power point on this voltage is $139.2 \mathrm{uW}$. Therefore power gain from this MPPT is $10.2 \mathrm{uW}$ for each solar cell, for 4 solar cells, the power gain from the MPPT is approximately $40.8 \mathrm{uW}$. Taking the MPPT power consumption into consideration, the $10.8 \mathrm{uW}$ power benefit from this MPPT is limited, it only accounts for a $1.5 \%$ increase in the power by using this MPPT approach.

From these considerations it appears that, in this type of low power system, the simplest way to track the maximum power point is by designing the energy storage and the load to make the operation voltage close to the $\mathrm{V}_{\mathrm{mpp}}$. The method will be introduced in next section.

\section{ENERGY StORAGE ELEMENTS}

As discussed in section 2.2, the voltage limited current source behaviour of the solar panel requires an energy storage element to separate the electric load and the solar panel. With an energy storage element, the solar panel based power supply will have voltage source-like behaviour, similar to the battery, hence, a more stable voltage output.

Three types of energy storage elements are frequently used in energy harvest systems, namely, Nickel Metal Hydride rechargeable battery, Lithium ion rechargeable battery and electrochemical double layer capacitors (also known as supercapacitors or ultra-capacitors). There are several significant different characteristics between these storage elements. (i) The super-capacitors have a lower energy density (energy/weight) than $\mathrm{NiMH}$ and Li-ion rechargeable battery, 
typically $20 \mathrm{Wh} / \mathrm{kg}$ compared to $70 \mathrm{Wh} / \mathrm{kg}$ and $160 \mathrm{Wh} / \mathrm{kg}$, respectively [17]. But the supercapacitors have higher power density (energy/time) than the rechargeable battery. The higher power density allows the supercapacitor be charged more quickly than the rechargeable batteries. (ii) The supercapacitors have a much higher number of charge-discharge cycles than any current battery technologies. Normally, it has over one million full charge-discharge cycles (deep cycles) compared to a rechargeable battery's less than 1000 cycles. (iii) Super-capacitors typically have a larger leakage current than rechargeable batteries. The high self-discharge rate is the main constraint for the supercapacitors' long term deployment. (iv) The rechargeable battery's charging circuits are more complicated than the supercapacitors'. The increase of the system complexity is more significant in solar panel based harvesters, since the solar panel's intrinsic voltage limited current source characteristics makes it difficult to charge a stable current input to the batteries, as is required. In addition, the Li-ion battery also requires a deep-discharge protection circuit, further increasing the system complexity.

Based on these features, supercapacitor type storage is selected as the energy storage system. When choosing a supercapacitor for this application, the ESR (Equivalent Series Resistance) of the capacitor is an important factor. In section 2, the power consumption pattern of the mote shows a large current is drawn during the active mode. A significant ESR therefore leads to voltage drop on the capacitor. Since the microcontroller's threshold voltage is $2.7 \mathrm{~V}$, if the voltage drop is larger than $0.6 \mathrm{~V}(3.3 \mathrm{~V}-2.7 \mathrm{~V})$, the microcontroller will be disabled, the data processing and transmission will fail. The small form factor Panasonic SD series EECS0HD104 has a $75 \Omega \mathrm{ESR}$, the active mode current is $33.1 \mathrm{~mA}$, and these give the voltage drop as high as $2.1 \mathrm{~V}$. To avoid this, a low ESR supercapacitor AVX bestcap supercapacitors are introduced [18]. This supercapacitor only has a $50 \mathrm{~m} \Omega$ ESR. When parallel connected with the Panasonic SD supercapacitor, the voltage drop during active mode is less than $0.9 \mathrm{mV}$.

In the previous section, it was described how the Perturband-Observe maximum power point tracking method's power consumption offsets the benefit from the MPPT; the fractional open circuit voltage method has a substantial tracking error in extra-low illuminance level and the tracker's power consumption also partly offsets the power gained from MPPT, as a result these two MPPT methods were not implemented, instead, only using a diode to prevent the reverse current flow into the solar panel.

Due to the direct connection of solar panel and supercapacitor and solar panel's inherent current source behaviour, the equivalent series resistance and the capacitance of the supercapacitors directly affect the voltage on the solar panel. Therefore, a pspice model is introduced to determine the capacitance and the ESR to make the solar panel operate close to its maximum power point voltage. This model uses Wolf and Rauschenbach's solar panel model [19], and the supercapacitor is considered as a series connected resistor and a ideal capacitor. Since the predicated light irradiance level for this application is between 100-1000lux, the average $V_{\text {mpp }}$ of 100lux, 200lux, 500lux and 1000lux is then chosen to be the average $\mathrm{V}_{\text {mpp }}$. The proposed $\mathrm{V}_{\text {mpp }}$ is then calculated at $2.92 \mathrm{~V}$. By using bisection method, repeatedly dividing the interval in half and selecting the subinterval, until it approaches the acceptable resolution, the supercapacitor's capacitance and resistance converge to $1.87 \mathrm{~F}$ and $30 \mathrm{~m} \Omega$. The panel operates at a close to maximum power point when the light irradiance is between 100lux and 1000lux, with an error between $-6 \%$ to $+7 \%$. The best fit is obtained at 500lux, with a $+2 \%$ approximation.

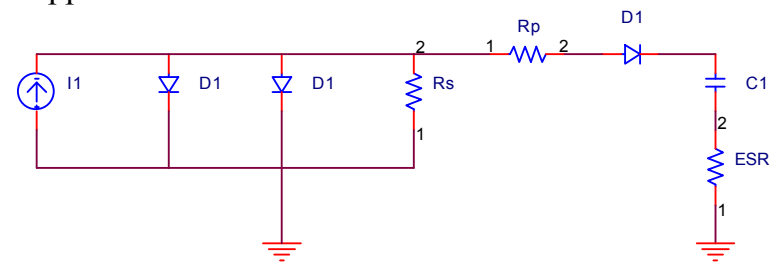

Fig.7 System Simulation Schematics

However, one drawback of this MPP optimization is that with the capacitance chosen so as to set the voltage for the MPPT, the charging and discharging time for the capacitor could not be optimized by scaling the capacitance of the supercapacitor.

\section{SYSTEM INTEGRATION AND PERFORMANCE}

The primary goal of our design is to optimize the system to obtain higher power conversion efficiency. Based on previous considerations on indoor solar irradiance and spectral response, a Schott Solar's ASI2Oi05 amorphous silicon solar panel is selected.

In this optimization, the capacitance of the supercapacitor bank is calculated at $1.87 \mathrm{~F}$ and the total equivalent series resistance is $0.03 \Omega$. Based on this capacitance and ESR, the design consists of a parallel connection of one AVX bestcap, and five Panasonic golden cap supercapacitors.

In addition, a voltage monitoring circuit is built to read the voltage level of the supercapacitor bank. Two switches are also incorporated, one to control the voltage level reading needed to send to Tyndall mote's microcontroller, the other to manually switch on/off the mote without disconnecting the mote from the solar energy harvesting module. Figure 8 shows the solar energy harvesting module that has been built.

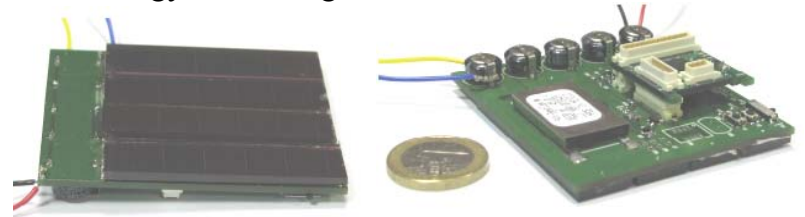

Fig8. Solar panel based energy harvester in system

The operational performance of the solar energy harvester module involved was tested by several experiments. The first experiment entailed charging the supercapacitor bank without mote operation. The aim of the experiment was to monitor the charging time and the supercapacitor bank's output voltage. Figure 9 plots the output voltage over time. The supercapacitor can be charged from $0 \mathrm{~V}$ to $3.1 \mathrm{~V}$ within 1.3 hours (4680 seconds). The pre-charged supercapacitor was then connected with the Tyndall mote in a bright office 
environment (bright fluorescent light, no sun light exposure) measured at 500lux, and tested during day time for 8 hours. The voltage of the supercapacitor bank over time is shown in Figure 9a. Each different part of the operation was measured at different times in the same day between $10 \mathrm{am}$ to $7 \mathrm{pm}$. The mote was able to correctly transmit data for the day and the voltage on the capacitors stabilised at $2.88 \mathrm{~V}$ and slightly increased during the two last measurements, due to a small increase in the illuminance (increase to 550lux) on the sample.
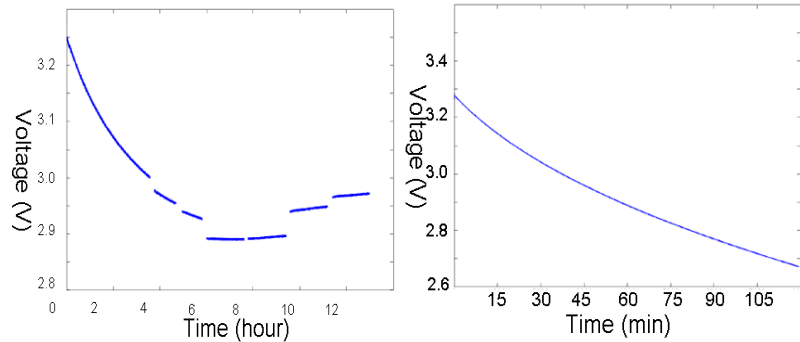

Fig9(a) Performance evulation of energy harvester (office environment) and 9(b) Performance evulation of energy harvester (dark)

The stabilized system then undertook an experiment in a dark environment, the power consumption of the mote being derived exclusively from the supercapacitor bank. The operation result was illustrated in Figure 9b. The normal operation of the mote lasted for 1.5 hours (5400 seconds), while the voltage of the capacitor bank gradually dropped to the mote's threshold voltage. The mote automatically entered deep-sleep mode and waited for power restoration.

These measured results show the indoor light powered wireless sensor module can operate autonomously during the presence of a typical indoor light illuminance. But it is unable to continuously operate in a total absence of light illuminance over a longer period of time. Since the aim of this deployment is to monitor the building environment during the working time, the light energy harvester could be considered as a reliable alternative of the conventional battery power supply.

\section{CONCLUSIONS}

Energy harvesting technologies show great potential as an important supplement for small wireless devices. Since one of the main constraints of the wireless devices is lack of an everlasting and maintenance free power supply, more and more researchers consider energy harvesting to be an essential part of future wireless devices. However, many design methodologies are insufficient and immature, especially in low energy input conditions.

This paper presents the environmental factors, components choices, design concept and main trade-offs in a solar panel based energy harvesting device design. A practical energy harvester module was designed and tested with a Tyndall $25 \mathrm{~mm}$ mote system, the performance was presented and evaluated. Autonomous operation, during presence of a typical indoor light illuminance, indicates the feasibility of indoor light energy harvesting for wireless sensor network applications.

Interesting further research problems exist in several areas, one of which is to prolong the operation time in dark environment by optimizing the energy storage elements, and also the possible design of a low power consumption maximum power point tracker for energy harvester.

\section{ACKNOWLEDGEMENT}

This work has been funded by Scientific Foundation Ireland through a research project ITOBO (398-CRP) and Enterprise Ireland research project BuildWise (RI2407).

\section{REFERENCES}

[1] Mohammad Rahimi, Hardik Shah, Gaurav Sukhatme, John Heidemann, and Deborah Estrin. "Studying the Feasibility of Energy Harvesting in a Mobile Sensor Network". In Proceedings of the IEEE International Conference on Robotics and Automation, Taipai, Taiwan, IEEE. May, 2003, pp. 19-24.

[2] Sensor Wireless INC. http://www.sensorwireless.com/

[3] Information and Communication Technology for the sustainable and Optimised Building Operation (ITOBO) Project. http://zuse.ucc.ie/itobo/

[4] D. Linden and T. Reddy. Handbook of Batteries, 3rd edit. McGrawHill, 2002.

[5] Torah, R., Beeby, S. P., Tudor, M. J., O'Donnell, T. and Roy, S. "Development of a Cantilever Beam Generator Employing Vibration Energy Harvesting", The 6th Int. Workshop on Micro and Nanotechnology for Power Generation and Energy Conversion Applications (PowerMEMS 2006), November 29th - December 1st 2006, Berkeley, USA

[6] M.Loreto, C.Cosim, L.Nestor, P.Markus, Spies.Peter, "Human Body Energy Harvesting Thermogenerator for Sensing Applications", Sensor Technologies and Applications, SensorComm2007. International Conference on 14-20 Oct. 2007 pp. $366-372$

[7] A. Virtuani, E. Lotter, M. Powalla, Influence of the light source on the low-irradiance performance of $\mathrm{Cu}(\mathrm{In}, \mathrm{Ga}) \mathrm{Se} 2$ solar cells, Solar Energy Materials and Solar Cells, Volume 90, Issue 14, 6 September 2006, pp. 2141-2149

[8] A 3-D Miniaturised Programmable Transceiver, Brendan O'Flynn, S. Bellis, K.Mahmood, M. Morris, G. Duffy, K. Delaney, C. O’Mathuna, Microelectronics International, Volume 22, Number 2, 2005, pp. 8-12

[9] Enocean self-powered wireless sensor, STM110c, http://www.enocean.com/en/enocean_modules1/

[10] R. Smith, C. Cifuentes, and D. Simon, Enabling Java ${ }^{\mathrm{TM}}$ for small wireless devices with Squawk and Spotworld, OOPSLA Workshop Bringing Software to Pervasive Computing, Oct 162005.

[11] Glunz, S.W. Dicker, J. Esterle, M. Hermle, M. Isenberg, et Al High-efficiency silicon solar cells for low-illumination applications, Photovoltaic Specialists Conference, 2002. Conference Record of the Twenty-Ninth IEEE, New Orleans, Louisiana, 19-24 May 2002, pp. 450- 453

[12] Arnulf Jager-Waldau, Status of thin film solar cells in research, production and the market, Solar Energy, Volume 77, Issue 6, Thin Film PV, December 2004, Pages 667-678

[13] Adolf Goetzberger, Christopher Hebling, HansWerner Schock, Photovoltaic materials, history, status and outlook, Materials Science and Engineering: R: Reports, Vol 40, Issue 1, 1 January 2003, Pg 1-46

[14] Texas Instruments, TPIC74100ql, Buck/Boost Switch Regulator,http://focus.ti.com/docs/prod/folders/print/tpic74100-q1.html

[15] Atmel, Atmega128L, Atmel AVR microcontroller, http://www.atmel.com/dyn/products/product card.asp?part id=2018

[16] Vijay Raghunathan; Kansal, A.; Hsu, J.; Friedman, J.; Mani Srivastava, "Design considerations for solar energy harvesting wireless embedded systems," Information Processing in Sensor Networks, 2005. IPSN 2005, pp. 457-462, 15 April 2005

[17] J.-M. Tarascon and M. Armand, Issues and challenges facing rechargeable lithium batteries, Nature 414, 15 November 2001, pp..359-367

[18] BestCap ${ }^{\circledR}$ Ultra-Low ESR High-Power Pulse Supercapacitor, AVX,http://www.avx.com/prodinfo_catlis asp?ParentID $=42$

[19] M. Wolf and H. Rauschenbach, "Series resistance effects on solar cell measurements," Adv. Energy Conv., vol. 3, pp. 455-479, 1963 